\title{
Risk of major adverse perinatal outcomes in women with eating disorders
}

\author{
NADIA MICALI, EMILY SIMONOFF and JANET TREASURE
}

\section{Background Low birth weight, prematurity and higher miscarriage rates have previously been reported in women with eating disorders.}

\begin{abstract}
Aims To determine whether women with a history of eating disorders are at higher risk of major adverse perinatal outcomes.
\end{abstract}

\begin{abstract}
Methods Adjusted birth weight, preterm delivery and miscarriage history were compared in those with a history of eating disorders (anorexia nervosa $(n=171)$, bulimia nervosa $(n=199)$ and both $(n=82))$ and those with other $(n=1166)$ and no psychiatric disorders $(n=10636)$ in a longitudinal cohort study.
\end{abstract}

Results The group with bulimia nervosa had significantly higher rates of past miscarriages (relative risk ratio 2.0, $P=0.01$ ) and the group with anorexia nervosa delivered babies of significantly lower birth weight than the general population ( $P=0.0 \mathrm{I}$ ), which was mainly explained by lower pre-pregnancy body mass index. Preterm delivery rates were comparable across groups.

Conclusions Women with a history of eating disorders are at higher risk of major adverse obstetric outcomes. Antenatal services should be aware of this higher risk.

Declaration of interest None. Funding detailed in Acknowledgements.
Eating disorders are a common source of psychiatric morbidity in women of childbearing age (Van Hoeken et al, 2003). Previous studies on clinical samples have reported that women with anorexia nervosa, bulimia nervosa and women hospitalised for an eating disorder deliver lower birth weight and more preterm babies (Stewart et al, 1987; Brinch et al, 1988; Bulik et al, 1999; Waugh \& Bulik, 1999; Sollid et al, 2004). Higher miscarriage rates have also been reported in women with eating disorders, especially those with bulimia nervosa (Mitchell et al, 1991; Abraham, 1998; Bulik et al, 1999; Blais et al, 2000).

No studies to date have determined, in an epidemiologically representative sample, whether the effect on adverse pregnancy outcomes is specific to the eating disorders and their symptoms, rather than to any severe psychiatric disorder. Moreover, most studies on women with eating disorders have not taken into account the effect of other mediating factors that may affect perinatal outcomes. In this study we investigated the effect of a history of eating disorders on the outcome of pregnancy in a representative sample of the British population.

\section{METHOD}

\section{Sample}

The Avon Longitudinal Study of Parents and Children (ALSPAC) is a longitudinal, prospective study of women and pregnancy (Golding et al, 2001). All pregnant women living in the geographical area of Avon, UK, who were expected to deliver their baby between 1 April 1991 and 31 December 1992 were recruited. All women gave informed and written consent. It was estimated that $85-90 \%$ of those eligible took part. The sample has been shown to be representative of the British population.

There were 14663 women enrolled at the 9th week of pregnancy. Data were obtained on 14472 women via postal questionnaires. Women were excluded from the current study if they had not answered the questionnaire sent at approximately 12 weeks (2019). We only included singleton births in the study (12254), as babies from multiple pregnancies have different patterns of foetal growth and gestational length. At 12 weeks women were also asked whether they had any recent or past history of psychiatric problems, including depression, schizophrenia, alcoholism, anorexia nervosa, bulimia nervosa or any other psychiatric disorder. Their pre-pregnancy weight and height were also obtained. Socio-demographic data were obtained during pregnancy. At 18 weeks of gestation information was obtained on vomiting and the use of laxatives for weight loss prior to and during pregnancy. Data on smoking and alcohol intake before and during the first and second trimesters of pregnancy were obtained at two time-points during pregnancy. Body mass index (BMI) was calculated as pre-pregnancy weight/height squared.

\section{Outcomes}

Birth weight, outcome of pregnancy (live or stillbirth), gender of the baby and gestational age at birth were obtained from obstetric records. Birth weights were corrected for gestational age and gender. Preterm delivery was defined as birth before 37 weeks of gestation. Only pregnancies where clinical estimates of length of gestation based on ultrasonography agreed with mothers' dates (plus or minus 2 weeks) were included. Women were asked at 18 weeks about any previous miscarriages. The data were then categorised as none, one and two or more.

\section{Data analysis}

Parametric (one-way analysis of variance) and non-parametric tests were used as appropriate for group comparisons, after testing for normality. Bivariate linear regression models were used to test for predictors of continuous outcomes. Multinomial and binary logistic regression models examined predictors of categorical and binary outcomes respectively.

Potential covariates likely to influence outcomes were first tested in bivariate models and included in multivariate models when significant. The final model accounted for the main effects of each covariate. Factors considered to be possible mediators (Kraemer et al, 2001) of main effects were included in the multivariate 
model at a second stage. All analyses were performed using Stata version 8 for Windows. All statistical tests presented are two-tailed. Statistical significance was defined as $P<0.05$.

Although our sample was relatively big, the sizes of groups with eating disorders were variable and some groups were small (anorexia nervosa plus bulimia nervosa in particular) in relation to the 'general population' control sample. We were therefore concerned that differences in rarer outcomes might not be detectable when comparing groups with eating disorders and the reference group. Hence we carried out a power calculation and found that effect sizes of 0.3 in continuous outcomes could be detected with a power of $75-93 \%$ at the $5 \%$ significance level. Group differences in proportions for common outcomes could be detected with $92-99 \%$ power and group differences in proportions for uncommon outcomes (such as prematurity) could be detected with $63-99 \%$ power at the $5 \%$ significance level.

\section{Ethical approval}

The study was approved by the ethics committees of the Institute of Psychiatry and ALSPAC.

\section{RESULTS}

Women who were included in the current study $(n=12254)$ were divided into five groups: (a) $171(1.4 \%)$ who only endorsed the question 'Have you ever had anorexia nervosa?' (7 of these reported a recent episode); (b) 199 (1.6\%) who only endorsed the question 'Have you ever had bulimia nervosa?' (51 of these reported a recent episode); (c) $82(0.7 \%)$ who endorsed both questions; (d) 1166 (9.5\%) who reported having had schizophrenia, severe depression or other psychiatric disorders (including drug addiction and alcoholism) and formed the 'other psychiatric disorders' group; (e) 10636 who formed the 'general population' comparison group.

\section{Socio-demographic data}

Maternal age at delivery and ethnicity did not differ across the five groups (see Table 2). Women with other psychiatric disorders were less likely to be in full-time or part-time employment, or full-time education or training and were more likely to be multiparous than the general population sample. Women in the three eating disorder groups did not differ from the general population sample on parity or employment status. Women with a history of anorexia nervosa, anorexia nervosa plus bulimia nervosa and other psychiatric disorders were significantly more likely to have smoked during the first trimester of pregnancy. Women with other psychiatric disorders were significantly more likely to have smoked during the second trimester of pregnancy and drunk alcohol during the first trimester. All four clinical groups were less likely to be living with a partner than the 'general population' group.

\section{Eating disorders and related symptoms}

We compared BMI across the five groups and the proportions of women reporting past vomiting and laxative use for weight loss (Table 2). Women in the three eating disorder groups were significantly more likely to have used laxatives and selfinduced vomiting. Women with a history of anorexia nervosa and anorexia nervosa plus bulimia nervosa had a significantly lower mean BMI than the other groups (Table 2).

\section{Pregnancy outcomes}

Foetal deaths $(n=66)$ were excluded from these analyses. Women with a history of anorexia nervosa had 2 foetal deaths $(1.2 \%)$, those with bulimia nervosa and those with anorexia nervosa plus bulimia nervosa had none, those with other psychiatric disorders had $7(0.6 \%)$ and general population controls had $57(0.7 \%)$. Differences were not statistically significant.

\section{Birth weight}

We excluded 67 women who developed gestational diabetes because of high rates of macrosomia in this group. Rates of gestational diabetes were significantly higher in the group with anorexia nervosa plus bulimia nervosa ( 2 positive, $2.4 \%$, Fisher's exact $=17.9, P=0.01$ ) and that with other psychiatric disorders (16 positive, $1.4 \%)$ compared with the general population (48 positive, $0.5 \%$ ). Data were missing on birth weight for 148 babies.

Mean birth weights corrected for gender and gestational age were calculated for 11973 babies. The mean birth weight for babies born to women with a history of anorexia nervosa was $3340 \mathrm{~g}(95 \%$ CI

Table I Socio-demographic data

\begin{tabular}{|c|c|c|c|c|c|}
\hline & $\begin{array}{l}\text { Anorexia nervosa } \\
\qquad n=|7|\end{array}$ & $\begin{array}{l}\text { Bulimia nervosa } \\
\qquad n=199\end{array}$ & $\begin{array}{l}\text { Anorexia nervosa plus } \\
\text { bulimia nervosa } \\
\qquad n=82\end{array}$ & $\begin{array}{c}\text { Other psychiatric } \\
\text { disorders } \\
n=1166\end{array}$ & $\begin{array}{c}\text { General } \\
\text { population } \\
n=10636\end{array}$ \\
\hline Age at delivery, years: mean (s.d.) & $28.9(5.2)$ & $28.2(4.6)$ & $29.2(4.6)$ & $28(5.5)$ & $28.2(4.8)$ \\
\hline Multiparity, \% (OR, 95\% Cl) & $52.5(0.9,0.6-1.2)$ & $51.6(0.9,0.7-1.2)$ & $53.3(0.9,0.6-1.5)$ & $59.1(1.2,1.0-1.3)^{* * *}$ & 54.9 \\
\hline White ethnicity, \% (OR, 95\% Cl) & $96.2(0.6,0.3-1.4)$ & $97.4(0.9,0.4-2.2)$ & $98.8(1.9,0.3-13.8)$ & $98(1.2,0.8-1.9)$ & 97.6 \\
\hline Employment, \% (OR, 95\% Cl)' & $49.0(1.0,0.7-1.4)$ & $48.9(1.0,0.7-1.3)$ & $42.7(0.8,0.5-1.2)$ & $32.8(0.6,0.6-0.7)^{* * *}$ & 49.1 \\
\hline $\begin{array}{l}\text { Any smoking in first trimester, } \\
\%(\mathrm{OR}, 95 \% \mathrm{Cl})\end{array}$ & $27.8(1.4,1.0-2.0)^{*}$ & $26.2(1.3,0.9-1.8)$ & $39.5(2.4,1.5-3.8)^{* * *}$ & $40.2(2.5,2.2-2.8)^{* * *}$ & 21.4 \\
\hline $\begin{array}{l}\text { Any smoking in second trimester, } \\
\%(\mathrm{OR}, 95 \% \mathrm{Cl})\end{array}$ & $20.2(1.3,0.9-1.9)$ & $20.6(1.3,0.9-1.8)$ & $23.5(1.6,0.9-2.6)$ & $32.6(2.5,2.2-2.8)^{* * *}$ & 16.3 \\
\hline Has a partner, \% (OR, 95\% Cl) & $95.2(0.4,0.2-0.8)^{*}$ & $95.6(0.4,0.2-0.9)^{*}$ & $90.5(0.2,0.1-0.3)^{* * *}$ & $94.6(0.4,0.3-0.5)^{* * *}$ & 98.1 \\
\hline $\begin{array}{l}\text { Any alcohol use in first trimester, } \\
\%(\mathrm{OR}, 95 \% \mathrm{Cl})\end{array}$ & II.6 $(0.7,0.4-1.2)$ & $18.8(1.3,0.9-1.9)$ & $24.7(1.8,1.1-3.0)^{*}$ & $19.3(1.3,1.1-1.6)^{*}$ & 15.2 \\
\hline
\end{tabular}

I. Percentage in full-time/part-time employment or full-time education/training $v$. unemployed, housewives or retired.

$* P<0.05, * * * P<0.001$ v. general population. 


\begin{tabular}{|c|c|c|c|c|c|}
\hline & $\begin{array}{l}\text { Anorexia nervosa } \\
\qquad n=175\end{array}$ & $\begin{array}{l}\text { Bulimia nervosa } \\
\qquad n=199\end{array}$ & $\begin{array}{l}\text { Anorexia nervosa plus } \\
\text { bulimia nervosa } n=82\end{array}$ & $\begin{array}{l}\text { Other psychiatric } \\
\text { disorders } n=1166\end{array}$ & $\begin{array}{c}\text { General population } \\
\qquad n=10636\end{array}$ \\
\hline $\begin{array}{l}\text { Self-induced vomiting for weight } \\
\text { control, } \%\end{array}$ & $23.4^{* * *}$ & $56.3^{* * *}$ & $62.2^{* * *}$ & 4.0 & 3.6 \\
\hline Laxative use for weight control, \% & $25 * * *$ & $29.1 * * *$ & $55^{* * *}$ & 4.5 & 3.2 \\
\hline $\begin{array}{l}\text { Body mass index pre-pregnancy: } \\
\text { mean (s.d.) }\end{array}$ & $21.5(3.2)^{* *}$ & $23.1(4.3)$ & $21.5(3.0)^{*}$ & $23.1(4.2)$ & $22.9(3.8)$ \\
\hline
\end{tabular}

$* P<0.05, * * P<0.01, * * * P<0.001, v$ general population.

Table 3 Linear regression analysis of birth wright after stepwise adjustment for relevant covariates

\begin{tabular}{|c|c|c|c|c|}
\hline & $\begin{array}{l}\text { Anorexia nervosa } \\
\qquad n=159\end{array}$ & $\begin{array}{l}\text { Bulimia nervosa } \\
\qquad n=195\end{array}$ & $\begin{array}{l}\text { Anorexia nervosa plus } \\
\text { bulimis nervosa } n=78\end{array}$ & $\begin{array}{l}\text { Other psychiatric } \\
\text { disorders } n=110 \mathrm{I}\end{array}$ \\
\hline & \multicolumn{4}{|c|}{$\mathrm{B}(95 \% \mathrm{Cl}) \beta$} \\
\hline \multirow[t]{2}{*}{ Birth-weight' } & $-83.9(-151.9$ to -15.9$)$ & $14.3(-48.7$ to 77.4$)$ & $-2.6(-101.7$ to 96.5$)$ & $-33.5(-60.9 /-6.1)$ \\
\hline & $-0.018^{*}$ & 0.003 & 0.000 & $-0.018^{*}$ \\
\hline \multirow[t]{2}{*}{ Birth weight' adjusted for maternal factors } & $-75.1(-143.6$ to -6.5$)$ & $20.5(-42.4 / 83.5)$ & $-2.6(-101.7$ to 96.5$)$ & $-36.4(-64.0$ to -8.8$)$ \\
\hline & $-0.016^{*}$ & 0.004 & -0.000 & $-0.019 * *$ \\
\hline Birth weight' adjusted for maternal factors & $-64.5(-132.3 / 3.3)$ & $27.6(-34.5$ to 89.7$)$ & $8.8(-90.2$ to 107.9$)$ & $-4.4(-31.9$ to 23.2$)$ \\
\hline and smoking in the second trimester & $-0.013^{2}$ & 0.006 & 0.001 & -0.002 \\
\hline Birth-weight' ${ }^{1}$ adjusted for maternal factors, & $-40.7(-109.1$ to 27.8$)$ & II.5 (- 52.6 to 75.5$)$ & $23.9(-78.8$ to 126.6$)$ & $-2.7(-31.0$ to 25.5$)$ \\
\hline smoking in the second trimester and BMI & -0.009 & 0.003 & 0.003 & -0.001 \\
\hline
\end{tabular}

BMI, body mass index.

$* P<0.05, * * P<0.01$.

I. Adjusted for gestational age and gender of the baby.

2. $P=0.06 \mathrm{v}$. general population.

3272-3407); to women with bulimia nervosa $3439 \mathrm{~g}$ (3377-3502); to women with anorexia nervosa plus bulimia nervosa $3422 \mathrm{~g}$ (3323-3521); to women with other psychiatric disorders 3392g (3366-3413); and to the general population sample $3425 \mathrm{~g}$ (3416-3433). Babies of women with anorexia nervosa were significantly lighter than babies of control women, as were babies of women with other psychiatric disorders (overall $F(6,11966)=918.8$, $P<0.05$ ) (Table 3).

We studied the role of covariates known to influence birth weight, including maternal factors such as parity, maternal age, employment status, whether women had a partner and alcohol intake (a factor relating to the studied pregnancy). Alcohol intake, relationship status and employment status were not significantly related to the outcome and were not included in the final model.

Smoking in the first and second trimester, pre-pregnancy BMI, laxative use and self-induced vomiting in pregnancy were investigated as possible mediators of effect.
Laxative use and self-induced vomiting in pregnancy were not significantly related to birth weight in bivariate analyses. When maternal covariates (parity, maternal age) were included in the model, babies born to women with a lifetime history of anorexia nervosa were still significantly lighter than babies of control women $(B=-75.1$, $\beta=-0.016, \quad P=0.03$ ) (Table 3). When smoking in the second trimester was included in the model, a marginal difference remained for babies of women with anorexia nervosa compared with general population controls $(B=-63.5, \beta=-0.013$, $P=0.06$ ). When BMI pre-pregnancy was included in the model, the effect of maternal history of anorexia nervosa on birth weight disappeared.

\section{Preterm delivery}

Data for evaluation of preterm delivery were available on 12188 births. The rates of preterm delivery were: anorexia nervosa $6.5 \%$; bulimia nervosa $5.0 \%$; anorexia nervosa plus bulimia nervosa $4.9 \%$; other psychiatric disorders $5.8 \%$; general population
$4.8 \%$; with no group differences on logistic regression analysis. After controlling for ethnicity, maternal age, and parity, the group with other psychiatric disorders had significantly higher rates of preterm delivery compared with the general population (odds ratio 1.3, 95\% CI 1.0-1.8, $P=0.03$ ).

\section{Previous miscarriages}

Data on previous miscarriages were analysed in 11700 women. An initial multinomial logistic regression showed that women with bulimia nervosa, those with anorexia nervosa plus bulimia nervosa and those with other psychiatric disorders were significantly more likely to report previous miscarriages (Table 4). When adjusted for relevant covariates (lifetime smoking and alcohol use, age, parity), only women with a history of bulimia nervosa and of other psychiatric disorders remained significantly more likely to have a history of previous miscarriages than the general population. A trend remained for women with anorexia nervosa plus bulimia nervosa. 


\begin{tabular}{|c|c|c|c|c|}
\hline & $\begin{array}{l}\text { Anorexia nervosa } \\
\qquad n=159\end{array}$ & $\begin{array}{c}\text { Bulimia nervosa } \\
\qquad n=195\end{array}$ & $\begin{array}{l}\text { Anorexia nervosa plus } \\
\text { bulimia nervosa } n=79\end{array}$ & $\begin{array}{l}\text { Other psychiatric } \\
\text { disorders } n=1100\end{array}$ \\
\hline \multicolumn{5}{|l|}{ Any previous miscarriages } \\
\hline Unadjusted OR $(95 \% \mathrm{Cl})$ & I.2(0.9-I.8) & I.4* (I.I-I.9) & $1.7 *(1.1-2.7)$ & $1.7^{* * *}(1.5-1.9)$ \\
\hline Adjusted' OR (95\% Cl) & $1.0(0.7-1.6)$ & I.4*(I.I-2.0) & $1.4(0.9-2.4)$ & $1.6^{* * *}(\mathrm{I} .4-\mathrm{I} .8)$ \\
\hline \multicolumn{5}{|l|}{ Number of miscarriages } \\
\hline One, unadjusted OR $(95 \% \mathrm{Cl})$ & $1.0(0.7-1.6)$ & $1.2(0.8-1.8)$ & $1.2(0.6-2.2)$ & $1.5 * * *(1.3-1.7)$ \\
\hline Two or more unadjusted, OR $(95 \% \mathrm{Cl})$ & $1.5(0.8-2.8)$ & $2.0 * *(1.2-3.3)$ & $3.2^{* * *}(1.6-6.3)$ & $2.5^{* * *}(2.0-3.1)$ \\
\hline One, adjusted' OR $(95 \% \mathrm{Cl})$ & $0.9(0.6-1.5)$ & $1.2(0.8-1.8)$ & $1.0(0.6-2.0)$ & $1.4^{* * *}(1.2-1.7)$ \\
\hline Two or more, adjusted' RRR $(95 \% \mathrm{Cl})$ & $\mathrm{I} .4(0.7-2.7)$ & $2.1^{* *}(1.2-3.6)$ & $2.8 * *(1.4-5.8)$ & $2.2^{* * *}(1.7-2.8)$ \\
\hline
\end{tabular}

$\mathrm{OR}$, odds ratio; $\mathrm{RRR}$, relative risk ratio.

$* P<0.05, * * P<0.01, * * * P<0.001$ v. general population.

I. Adjusted for maternal age, parity, lifetime alcohol use (more than I glass of alcohol/week), lifetime smoking.

The same three groups of women were significantly more likely to have had two or more miscarriages compared with the general population. The difference remained after controlling for relevant covariates (Table 4).

\section{DISCUSSION}

\section{Main findings}

Women with a history of bulimia nervosa (with or without a history of anorexia nervosa) had an increased rate of lifetime miscarriages, as did women with a history of other psychiatric disorders. This persisted after controlling for potential covariates. Women with a history of anorexia nervosa were more likely to deliver babies of lower birth weight than control women, although weights were comparable to babies of women with other psychiatric disorders.

\section{Miscarriages}

Higher rates of miscarriage in women with bulimia nervosa have been reported previously (Mitchell et al, 1991; Morgan et al, 2006). A higher risk of miscarriage for women with current and past bulimia nervosa was reported in two studies (Abraham, 1998; Blais et al, 2000). Our results confirm these findings. Possible hypotheses include polycysitic ovary syndrome and leptin abnormalities (Morgan et al, 2006). Future research will need to address the issue of direct cause of miscarriages in women with bulimia nervosa and the exact physiology.

\section{Birth weight}

Previous studies have shown that women with current or past eating disorders have a higher risk of delivering lower birth weight babies (Stewart et al, 1987; Bulik et al, 1999; Sollid et al, 2004) and our study confirms this finding. However, we found that the lower birth weight of babies born to women with anorexia nervosa may be mediated by lower pre-pregnancy BMI and to a lesser extent by smoking in the second trimester of pregnancy. None of the previous studies has investigated the effect of either variable in a population with eating disorders. However, the effect of maternal weight pre-pregnancy on birth weight of offspring has been documented in population studies; low maternal weight at conception or delivery has been found to have a significant impact on perinatal outcomes, mainly birth weight and preterm delivery (Kaminsky et al, 1973; Wolfe et al, 1991; Cnattingius et al, 1998; Ehrenberg et al, 2003). It is likely that a low prepregnancy BMI is an indicator of poor maternal nutritional status during pregnancy, but we were not able to evaluate this in this study.

Previous studies have highlighted an increased risk for adverse perinatal outcomes in women with severe mental illness (Jablensky et al, 2005), but no previous study has compared women with eating disorders with women with other severe psychiatric disorders. In our study, smoking during the second trimester seemed to be mainly responsible for the low birth weight in women with other psychiatric disorders. This suggests that the mechanism for low birth weight might be different in women with other severe psychiatric disorders compared with women with anorexia nervosa.

\section{Preterm delivery}

Two previous studies of clinical samples have shown higher rates of prematurity in babies of women with eating disorders (Bulik et al, 1999, Sollid et al, 2004). Bulik et al (1999) relied on a small sample and self-report of premature birth. The study of Sollid et al (2004), although larger, was register-based and included only women who had been hospitalised for an eating disorder, which was likely to be severe. Recall and sampling differences might therefore partly explain the disparity of these findings with those of our study. Our study is in line with that of Franko et al (2001) who found no difference in rates of prematurity when comparing women with anorexia and bulimia nervosa. There is the possibility that this finding might be a result of a low power to detect differences in our sample. This finding needs replication.

\section{Strengths and limitations}

The strengths of the study include the use of data from a large longitudinal prospective community cohort. We were able to include a comparison group of women with psychiatric disorders other than eating disorders in addition to a general population control group. We were also able to take into account the role of several covariates relevant to the outcomes.

The main weakness of this study is that women were classified according to self-report of lifetime anorexia nervosa or bulimia nervosa or both. It is uncertain how accurate this classification is in terms of psychiatric classificatory systems. However, the availability of rates of lifetime eating disorder behaviours and BMI prepregnancy lends weight to self-reported diagnoses. The prevalence of eating disorders in this sample was $3.7 \%$. According to estimates of the prevalence of eating disorders in women of child-bearing age 
(Striegel-Moore et al, 2006), the prevalence of anorexia nervosa is between 0 and $1.5 \%$ and that of full-syndrome bulimia nervosa between is 0.4 and $0.8 \%$. When partial syndromes are included the prevalence rate of eating disorders reaches about $5 \%$. The prevalence of anorexia nervosa in our sample is $1.4 \%$ and that of bulimia nervosa $1.6 \%$. It is therefore likely that a proportion in these two groups might have had an eating disorder not otherwise specified or a milder eating disorder compared with clinical samples. The current study is therefore likely to have underestimated rather than overestimated the rates of adverse perinatal outcomes in women with eating disorders.

Another limitation of the study is that weights and heights pre-pregnancy were also obtained by self-report. Moreover, we were not able to determine the temporal relationship between previous miscarriages and the course of bulimia nervosa. The sample did not have sufficient power to determine whether rare complications such as foetal deaths were more common in women with anorexia nervosa, although there was a trend in this direction.

\section{Implications}

Our results, together with previous reports in the literature, suggest that maternal eating disorders are associated with higher risk of some obstetric complications. This is extremely relevant to the prevention of adverse foetal outcomes. Moreover, the extent to which perinatal complications are predictors of later psychiatric disorders is still unclear. We found that women with eating disorders have similar rates of major adverse perinatal outcomes to women with other psychiatric disorders, although some of the causal factors implicated might differ. Women with bulimia nervosa are at higher risk of miscarriage. Future research will need to clarify the exact mechanism.

Women with a history of anorexia nervosa should be informed when planning a pregnancy that good general health includes having a healthy BMI as well as smoking cessation. Previous studies suggest that the association of smoking with high levels of body image distortion, and the role of smoking in weight control are relevant to women with and without eating disorders (George \& Waller, 2005; John et al, 2006). If this is so, the link with body image and weight control may need to be considered when counselling women about smoking cessation in pregnancy.

NADIA MICALI, MD, MRCPsych, Department of Child and Adolescent Psychiatry and Eating Disorders Research Unit, Institute of Psychiatry, King's College London; EMILY SIMONOFF, MD, FRCPsych, Department of Child and Adolescent Psychiatry, Institute of Psychiatry, King's College London; JANET TREASURE, PhD, FRCP, FRCPsych, Eating Disorders Research Unit, Department of Academic Psychiatry (Guy's Hospital), King's College London and ALSPAC, Department of Pediatric and Perinatal Epidemiology, University of Bristol, Bristol, UK

Correspondence: Dr Nadia Micali, Department of Child and Adolescent Psychiatry, Institute of Psychiatry, King's College London, Box 085, De Crespigny Park, London SE5 8AF, UK. Email: N.Micali@iop.kcl.ac.uk

(First received 14 December 2005, final revision 10 October 2006, accepted 8 November 2006)

Experts agree that women should be counselled to delay pregnancy until the eating disorder is in complete remission (Sollid et al, 2004). Advising women with eating disorders on possible effects of the disorder on fertility and the possibility of adverse outcomes in their offspring could be important for motivating women to implement changes in their behaviour.

\section{ACKNOWLEDGEMENTS}

We thank Dr Sam Leary, ALSPAC, for her help with the data, the women and children who took part in this study, the midwives and the whole ALSPAC team. The study was funded by the National Alliance for Schizophrenia and Depression and the Psychiatry Research Trust. The UK Medical Research Council, the Wellcome Trust and the University of Bristol provided core support for ALSPAC.

\section{REFERENCES}

Abraham, S. (1998) Sexuality and reproduction in bulimia nervosa patients over 10 years. Journal of Psychosomatic Research, 44, 491-502.

Blais, M. A., Becker, A. E., Burwell, R. A., et al (2000) Pregnancy: outcome and impact on symptomatology in a cohort of eating-disordered women. International Journal of Eating Disorders, 27, 140-149.

Brinch, M., Isager, T. \& Tolstrup, K. (1988) Anorexia nervosa and motherhood: reproduction pattern and mothering behavior of 50 women. Acta Psychiatrica Scandinavica, 77, 611-617.

Bulik, C. M., Sullivan, P. F., Fear, J. L., et al (1999) Fertility and reproduction in women with anorexia nervosa: a controlled study. Journal of Clinical Psychiatry, 60, 130-135

Cnattingius, S., Bergstrom, R., Lipworth, L., et al (1998) Prepregnancy weight and the risk of adverse pregnancy outcomes. New England Journal of Medicine, 338, $147-152$

Ehrenberg, H. M., Dierker, L., Milluzzi, C., et al (2003) Low maternal weight, failure to thrive in pregnancy, and adverse pregnancy outcomes. American Journal of Obstetrics and Gynecology, 189, 1726-1730.

Franko, D. L., Blais, M. A., Becker, A. E., et al (200I) Pregnancy complications and neonatal outcomes in women with eating disorders. American Journal of Psychiatry, 158, 146I-1466.
George, A. \& Waller, G. (2005) Motivators for smoking in women with eating disorders. European Eating Disorders Review, 13, 417-423.

Golding, J., Pembrey, M. \& Jones, R. (200I)

ALSPAC - the Avon Longitudinal Study of Parents and Children. I. Study methodology. Paediatric and Perinatal Epidemiology, 15, 74-87.

Jablensky, A., Morgan, V., Zubrick, S., et al (2005) Pregnancy, delivery and neonatal complications in a population cohort of women with schizophrenia and major affective disorders. American journal of Psychiatry, |62, 79-91.

John, U., Meyer, C., Rumpf, H.-J., et al (2006) Psychiatric comorbidity including nicotine dependence among individuals with eating disorders criteria in an adult general population sample. Psychiatry Research, 14I, 7l-79.

Kaminsky, M., Goujard, J. \& Rumeau-Roquette, C. (1973) Prediction of low birth weight and prematurity by a multiple regression analysis with maternal characteristics known since the beginning of pregnancy. International Journal of Epidemiology, 2, 195-204.

Kraemer, H. C., Stice, E., Kazdin, A., et al (200I) How do risk factors work together? Mediators, moderators, and independent, overlapping, and proxy risk factors. American Journal of Psychiatry, I58, 848856.

Mitchell, J. E., Specker, S. M. \& de Zwaan, M. (199I) Comorbidity and medical complications of bulimia nervosa. Journal of Clinical Psychiatry, 52 (suppl.), 13-20.

Morgan, J. F., Lacey, J. H. \& Chung, E. (2006) Risk of postnatal depression, miscarriage, and preterm birth in bulimia nervosa: retrospective controlled study. Psychosomatic Medicine, 68, 487-492.

Sollid, C. P., Wisborg, K., Hjort, J., et al (2004) Eating disorder that was diagnosed before pregnancy and pregnancy outcome. American Journal of Obstetrics and Gynecology, 190, 206-210.

Stewart, D. E., Raskin, J., Garfinkel, P. E., et al (1987) Anorexia nervosa, bulimia, and pregnancy. American Journal of Obstetrics and Gynecology, 157, 1194-1198.

Striegel-Moore, R., Franko, D. \& Ach, E. (2006) Epidemiology of eating disorders: an update. In Annual Review of Eating Disorders (eds S. Wonderlich, J. E. Mitchell, M. de Zwaan, et al). pp. 65-80. Radcliffe.

Van Hoeken, D., Seidell, J. \& Hoek, H. (2003) Epidemiology. In Handbook of Eating Disorders (eds J. Treasure, U. Schmidt \& E. van Furth), pp. II-34. Wiley.

Waugh, E., Bulik, C.M. (1999) Offspring of women with eating disorders. International Journal of Eating Disorders, 25, 123-133.

Wolfe, H. M., Zador, I. E., Gross, T.L., et al (199I) The clinical utility of maternal body mass index in pregnancy. American Journal of Obstetrics and Gynecology, 164, 13061310 